\title{
An Adaptive Long-Term Bus Arrival Time Prediction Model with Cyclic Variations
}

\author{
Prakhar Balasubramanian \\ K. Ramachandra Rao \\ IIT Delhi
}

\begin{abstract}
Real-time bus arrival information systems at transit stops can be useful to passengers for efficient trip planning and reducing waiting times. The accuracy of such systems depends upon the ability of the model to account for variations in the data series and to adjust according to changing traffic conditions. Many of the existing studies on passenger information systems have modeled the system based on stationary relations, not taking into account the cyclic variations in data, which is often suitable for demonstration purposes but not for long-term implementation. The present study models the changing relationships using Double-Seasonal Holt-Winter's Exponential Smoothing approach, which allows for self-updating parameters at four levels. It accounts for both long-term and short-term seasonal fluctuations in data while maintaining the dynamic treatment of real-time bus information. The model also takes into account delays using the real-time running information of the bus and incorporates it into subsequent forecasts for better accuracy. Real-time data from GPS transmitters in buses were used for validation of the proposed model. The results show that the proposed model performs better than the currently-used elementary field methods and is able to forecast bus travel times with a reasonable accuracy.
\end{abstract}

\section{Introduction}

Traffic congestion is a problem that affects transportation networks in many major cities in terms of reduced mobility and system reliability. In practice, particularly in transit-oriented cities, passengers often are faced with a choice between different modes of transport-for instance, buses and metro rail in Delhi. Bus systems often suffer a disadvantage, as rail and other transportation networks often have fixed schedules that are fairly accurate.

In densely-populated cities (e.g., Mumbai, Delhi, and Kolkata), with heavy passenger demands, real-time Passenger Information System (PIS) on arrival times of buses can be 
very helpful to the commuters. This can prove to be useful, particularly at major bus stops with large numbers of transfer passengers to other modes (rail, BRT etc.). The availability of real-time transit arrival information can help in efficient trip planning and making smart choices for travel. Further, real-time information can attract potential transit users to the system due to the inherent advantages of the system.

In recent times, advances in the field of Information and Communication Technologies (ICT) have opened up new avenues for integrating real-time information in passenger information systems. Recently, transit agencies also have started implementing ICT-related technologies in their operations to help in improving operational efficiency and the quality of information (Yu 2011).

Such advanced systems are lacking in most Indian cities, and the few that have been implemented are very elementary in nature and have focused largely on technology demonstration. However, the reliability of such systems greatly depends on the ability of the system to account for different variations in the data series and to adjust to dynamic traffic conditions. Hence, there is an urgent need to develop a robust real-time bus arrival time prediction model that can be applied under dynamic Indian conditions (Vanjakshi et al. 2009; Ramakrishna et al. 2006; Padmanaban 2010)

\section{Literature Review}

In the recent past, various sophisticated techniques and algorithms have been employed to forecast bus travel time or arrival time by using Global Positioning Systems (GPS)or bus triangulation data. These may be categorized as elementary (non-recursive) or advanced (recursive algorithms) based on the way they process the input data.

\section{Elementary Algorithms}

A variety of elementary approaches have been applied in the past studies to forecast travel times. Some of these include:

- Simple averages

- Linear regression

- Non-parametric regression (Zhang and Rice 2003)

- k-Nearest Neighbor (k-NN) (You and Kim 2000; Smith et al. 2002; Chang et al. 2010; Park et al. 2007; Baker and Nied, 2013)

These elementary algorithms process the whole data set after every new observation and often are computationally inefficient in practical applications.

\section{Advanced Algorithms}

\section{Moving Averages Models}

This model assumes that the forecast for period $i+1$ is the average of observations in the previous $n$ periods.

$$
F_{i+1}=\sum_{k=i-n}^{i} \frac{T_{k}}{n}
$$


The value of $n$ is chosen based on the nature of the data series. For a relatively stationary data series, a large value of $n$ is desirable. For a data series which is volatile, a small value of $n$ is chosen.

\section{Kalman Filter}

Kalman filter is an efficient recursive procedure that estimates the future states of dependent variables using a linear quadratic estimation model. It uses modern control theory to apply state space representations in the prediction scheme. Several studies have been carried out in the past using the Kalman Filter for bus travel time predictions using three components-a track, a filter, and a predictor (Chien and Kuchipudi 2003; Shalaby and Farhan 2004; Cathey and Dailey 2003; Son et al., 2004).

The predictor-corrector form of the Kalman filter that is used to calculate the filtered estimate is as follows:

$$
\hat{x}_{i j}(k \mid k)=\hat{x}_{i j}(k \mid k-1)+K_{i j}(k) \tilde{y}_{i j}(k \mid k-1)
$$

Where for the dynamic model $\tilde{y}_{i j}(k \mid k-1)$ is the measurement residual and is defined as:

$$
\tilde{y}_{i j}(k \mid k-1)=y_{i j}(k)-\hat{x}_{i j}(k \mid k-1)
$$

$K_{i j}(k)$ is the Kalman gain of arc $(i, j)$ at time $k$, which is specified by the following set of equations where $r_{i j}(k)$ is the measurement error in the system:

$$
K_{i j}(k)=\frac{\operatorname{cov}\left(x_{i j}(k), \tilde{y}_{i j}(k \mid k-1)\right)}{\operatorname{var}\left(\tilde{y}_{i j}(k \mid k-1)\right)}=\frac{\operatorname{var}\left(\tilde{x}_{i j}(k \mid k-1)\right)}{\operatorname{var}\left(\tilde{x}_{i j}(k \mid k-1)\right)+r_{i j}^{2}(k)}
$$

\section{Support Vector Machine Models (SVM)}

SVM is a learning algorithm that maps the input-output relationship in a non-linear system using statistical learning theory. In addition, SVM solves the model as a quadratic programming problem with linear constraints, thus always giving a unique and globally optimal solution. Therefore, SVM shows a high general performance by resisting the over-fitting problem (Richman and Parks 1998; Cristianini and Shawe-Taylor 2000; Vapnik 1999, 2000; Bin et al. 2006; Yu et al. 2010).

\section{Exponential Smoothing (ES) Models}

Simple exponential smoothing (SES) models are infinite order weighted averages and are used to predict the future state recursively based on the following relations:

$$
\begin{gathered}
s_{0}=x_{0} \\
s_{t}=\alpha x_{t}+(1-\alpha) s_{t-1}
\end{gathered}
$$

Where $\alpha$ is the weight assigned to the most recent observation and a weight (1- $\alpha$ ) assigned to combined past observations. This factor is chosen to achieve the best fit among the actual and the forecasted values. As a rule of thumb, if the data series is relatively stable, then a small value of the weight is used; a large value is used if the data series is volatile.

Although a very powerful prediction tool, SES models are based on the premise that the level of time series should fluctuate about a constant or change little over time. Li et 
al. (2008) used an Adaptive Simple Exponential Model (ASES) that could detect bias in forecasts and give an indication to re-calibrate the parameters. An ASES model was developed for highway travel time prediction using Kalman Filter to update the parameter weights (Liu et al. 2012). Also, various models have been developed to incorporate multiple factors into the forecasts. Holt-Winter's ES model uses three smoothing factors to forecast a time series with a linear time trend and single order cyclic variations. A double seasonal Holt-Winter's method was also used to predict short-term electricity demand (Miranda et al. 2006). This method is discussed further in subsequent sections.

\section{Artificial Neural Network (Effects across Space)}

An ANN model is used to find solutions for complex non-linear models by stimulating the intelligent capabilities of human brain. It tries to model the interaction between neurons and the connections by assigning weights. Using this interconnected link approach, it is able to model complex relationships and find patterns in the data series. Its implementation comprises two phases: the learning phase used to train the model and assign weights, and the recalling phase used to apply the weights assigned in the learning phase. Several studies in the past have employed the link-based to the stop-based ANN model to forecast bus arrival time (Chien et al. 2002; Chen et al. 2004; Jeong and Rilett 2004). Mazloumi et al. (2009) developed two models based on travel time data obtained from GPS traces of buses and traffic signal saturation flow data to predict the bus travel time using ANNs.

\section{Shortcomings of Current Models}

Many studies have been carried out on forecasting bus travel/arrival time for a single bus route. Most of the previous studies used some factors related to road traffic (e.g., traffic speed and volume) for modeling the system. However, very few studies accounted for cyclic fluctuations in data. Traffic data are influenced by several factors such as time of day and day of the week and might show an upward trending pattern in the long run. The arrival time prediction model can be improved by integrating these inputs into an adaptive prediction scheme. Also, most of the studies have broken down the travel route into different segments and then treated these as independent for the purpose of prediction. However, correlation among different segments can provide useful insights about the congestion state of the route and can be useful to improve prediction accuracy as bus progresses along the route.

Moreover, none of the studies have accounted for the changing nature of the traffic characteristics in both the short and long run. In the long run, the average traffic is expected to grow, resulting in an overall increase in travel time; in the short run, the dependence/ interaction of traffic streams across different periods may change according to time. The current models, once initialized and validated, assume functions that are stationary and do not change with time. Although these can successfully model and forecast travel times in the short run for research/demonstration purposes, the need for a more stable adaptive system for successful field implementation is evident.

\footnotetext{
${ }^{1}$ Long-term data cover all cyclic fluctuations-for instance, a week if only the daily fluctuations are being modeled or a year if seasonal fluctuations are being modeled. Short-term data are a subset of long-term data, which are insufficient to capture the entire cycle of fluctuations.
} 


\section{Objective and Scope}

The focus of this study was to improve forecasting in uncertain and dynamic environments by developing techniques that can be easily implemented in real time using robust and reliable methodologies. The study aims to achieve the following objectives:

- To arrive at a reasonable travel time estimate based on the real-time running information of the bus taking into account variations in traffic conditions on different times of the day and on different days of the week.

- To develop a computationally-efficient model that can perform the above tasks and also update (correct) itself automatically to adjust to changing traffic conditions.

- To implement and test the model on data from an existing route.

- To evaluate the performance of the proposed model with respect to the actual observed travel times and with other existing forecasting algorithms in use.

\section{Data Collection}

The route chosen for this study was Delhi Transport Corporation (DTC) bus Route 78 in New Delhi, India. This is one of the important routes in the city, with a length of approximately $20 \mathrm{~km}$ and a travel time of 40-60 minutes. The route comprises 15 bus stops connecting Azadpur Bus Terminal to Inderpur via Wazirpur, Punjabi Bagh, and Naraina, all of which are important and relatively crowded bus stops. Table 1 shows the details of the bus stops along with their relative distances from the starting bus stop.

TABLE 1.

Bus Stops on DTC Route 78

\begin{tabular}{|c|l|c|c|}
\hline Stop No. & \multicolumn{1}{|c|}{ Stop Name } & Distance between Stops $(\mathbf{k m})$ & Cumulative Distance (km) \\
\hline 1 & Azadpur Terminal & 0.00 & 0.00 \\
\hline 2 & Shalimar Bagh & 1.30 & 1.30 \\
\hline 3 & Ashok Vihar & 0.40 & 1.70 \\
\hline 4 & PerambariPul & 0.75 & 2.45 \\
\hline 5 & Wazirpur Depot & 0.70 & 3.15 \\
\hline 6 & Brittania Factory & 1.70 & 4.85 \\
\hline 7 & Punjabi Bagh & 3.80 & 8.65 \\
\hline 8 & Rampura & 2.20 & 10.85 \\
\hline 9 & Zakhira & 1.20 & 12.05 \\
\hline 10 & Swatantra Mills & 0.24 & 12.29 \\
\hline 11 & Moti Nagar & 2.60 & 14.89 \\
\hline 12 & Sp. Depot & 1.80 & 16.69 \\
\hline 13 & Naraina Depot & 0.85 & 17.54 \\
\hline 14 & Loha Mandi & 1.00 & 18.54 \\
\hline 15 & Inderpurji Colony & 0.60 & 19.14 \\
\hline & & & \\
\hline
\end{tabular}

For the present study, GPS data were provided by Delhi Integrated Multimodal Transit System (DIMTS), which was recorded by GPS units placed in the buses along the given route. A total of 30 days' data were available for the study (January 1-31, 2013). The GPS 
unit was inbuilt with a general packet radio service (GPRS) modem, which sent the location details to the remote server at a time interval of 10 seconds. The same objective also has been achieved using a GPS-enabled smart phone application (Biagioni et al. 2010). Zhou et al. (2014) demonstrated the use of crowd-sourced smart phones for collecting data and helped predict the expected arrival times of buses. This method seems interesting, given the fact that it is based on commuter participation.

\section{Methodology}

Forecasting travel time is a complex method. In this study, the process was divided into the following steps:

- Step 1 - Break down the route into smaller segments, usually between two bus stops. These smaller segments are subsequently treated as independent units.

- Step 2 (variations across time) - Forecast travel time (or speed, etc.) for each segment using an appropriate forecasting algorithm (Holt-Winter's Exponential Smoothing in the present case)

- Step 3 (variations across space) - Combine the travel time estimates from different segments with the spatial state of the route to account for the current traffic conditions.

- Step 4-Combine the above with the current status of the bus to predict arrival time.

\section{Prediction Framework}

\section{Nature of Traffic Data}

Traffic patterns across the route follow complex patterns depending upon different inputs, which can be difficult to account for. The present study accounted for the following to approximate for the variations in the data series, which can account for long-term variations in the data:

- Level Component - constant component of traffic/travel time

- Linear Time Trend - linearly increasing slope in traffic demand/travel time

- Hourly Variations - depending upon time of day

- Daily Variations - depending upon day of week.

Thus, the model recognizes the time-related variations in data series and correspondingly splits the space into corresponding factors while forecasting. In the study, the data series was directly dealt in terms of travel time, which automatically incorporated the variations in the traffic mix and other factors in different states. Traffic conditions were assumed to remain consistent in any given state.

\section{Forecasting Algorithm}

The study used the Double-Seasonal Holt-Winter's Exponential Smoothing model to carry out the prediction across segments. It is time series based on self-learning recursive algorithm based on non-linear relations that update its parameters based on real-time 
data inputs. It is a relatively new statistical model that, until now, has been tested to forecast highly seasonal fluctuations in time series data. The model allows for the above cyclic variations in the data series, which makes it suitable for the traffic data. Despite being computationally efficient, it is an infinite memory model; each forecast carries information about all of the past information even though the weights follow an exponentially decreasing trend, thus automatically discarding irrelevant informant from distant past.

\section{Estimate Function}

The algorithm assigns the following form to the estimate function:

$$
D_{t}=\left(S_{t}+G_{t}\right) \cdot d_{t} \cdot h_{t}+e_{t}
$$

Where,

$$
\begin{aligned}
& D_{t}=\text { estimated variable; bus travel time in the current case } \\
& S_{t} \quad=\text { level component } \\
& G_{t}=\text { slope/trend component } \\
& d_{t} \quad=\text { daily seasonal factor } \\
& h_{t} \quad=\text { hourly seasonal factor } \\
& e_{t} \quad=\text { random error component }
\end{aligned}
$$

\section{Linear Model Parameters}

$S_{t}$ and $G_{t}$ represent the intercept and components of the travel time function. This basically represents the change in traffic conditions over longer periods of time when the travel time is expected to increase due to increase in traffic on the road. In the short run, it represents average travel time across the route after filtering out the corresponding cyclic variations.

\section{Cyclic Variations}

The model has the linear component superimposed by two multiplicative seasonal factors that take into account the variations in traffic: hourly $\left(h_{t}\right)$ and daily $\left(d_{t}\right)$. Correspondingly, the data set has been split into eight two-hour intervals throughout the day and one hourly factor allotted to each period. Similarly, there are seven different daily factors, one for each day of the week.

First, the data are split into their different components and with different information filtered out, which are then combined to arrive at a forecast (see Figure 4). Thus, the model captures variations at four different levels in real time.

\section{Real-Time Updating of Parameters}

The model follows a two-stage filter-predictor approach to update its parameters:

- Filter (Stage 1) - The data from the actual travel time are de-seasonalized, corrected for time trend, and given a weight, $\alpha$. This is done by using the observed data and the most recent values of all other parameters to arrive at an estimate of the observed value of the parameter. Thus, the filter estimates the vehicle's dynamic state based on real-time observation. 
- Predictor (Stage 2) - The previous value of the parameter being updated is assigned a weight $(1-\alpha)$. Since these past data are a weighted average of all past information, the updated parameter is also a weighted average of all past data points.

The following relationships show how the parameters are updated in real time. Each factor uses its own weight to update itself after every new observation.

\begin{tabular}{|l|c|}
\hline Level & $S_{t}=\alpha \cdot \frac{D_{t}}{d_{t-s 1} \cdot h_{t-s 2}}+(1-\alpha)\left[S_{t-1}+G_{t-1}\right]$ \\
\hline Time-Trend & $G_{t}=\beta\left[S_{t}-S_{t-1}\right]+(1-\beta) G_{t-1}$ \\
\hline Hourly & $h_{t}=\delta \frac{D_{t}}{S_{t} \cdot d_{t}}+(1-\delta) h_{t-n_{2}}$ \\
\hline Forecast & $F_{t+n}=\left(S_{t}+n \cdot G_{t}\right) \cdot d_{t+n-n_{1} \cdot n_{2}} \cdot h_{t+n-n_{2}}$ \\
\hline
\end{tabular}

Where $n_{1}$ and $n_{2}$ represent the number of days in the week and the number of time intervals in the day defined in the model. In the current simulation, $n_{1}=7$ and $n_{2}=8$.

The weights are chosen depending upon the sensitivity of the data series to changes. Optimal values of these parameters are worked out by minimizing the mean squared errors on the validation data set. Given the current data series, it is intuitive to feel that these parameters should have a low value, as traffic conditions are expected to stay stable during one month observations of the data.

\section{Initialization of the Model}

Since the model is based on recursive relationships, it needs starting estimates of the parameters. These can be worked out using the following approach:

- A regression was carried out on the first week of data. Seasonality averages out to 1 ,and the slope and the intercept of the regression gives an initial estimate of the respective components of the demand function. Thus, we obtain starting estimates of $S_{t}$ and $G_{t}$.

- Divide the observed values by these calculated linear components to get estimate of $h_{t} \cdot d_{t}$.

- To calculate $d_{t}$, take average of all the observations for that particular day. The hourly factor averages out to 1 , and $d_{t}$ remains constant.

$$
d_{t}=\frac{\sum_{k=n_{2} t+1}^{n_{2} t+n_{1}} h_{k} \cdot d_{k}}{n_{2}}
$$


- To calculate $h_{t}$, take the average of all observations for that particular period for all days of the week. $h_{t}$ remains constant, and daily factor averages out to 1 . Thus, we have a starting estimate of $h_{t}$.

$$
h_{t}=\frac{\sum_{k=0}^{n_{1}-1} h_{n_{2} k+t} \cdot d_{n_{2} k+t}}{n_{2}}
$$

With the initial parameter estimates for the first cycle ( $t=0$ to 52$)$ determined as above, the estimates can be recursively updated through time to estimate the system state at any given time.

\section{Computational Efficiency}

The relationships used in the proposed model require a fixed number of mathematical operations to be carried out on the most recent observation to update the estimation of the system state, which is independent of the volume of the past data. Also, the model requires only past one cycle of data to be known to update itself, which is independent of the forecasting horizon or the volume of past data available. Once initialized, the model has a time and space complexity of $\Theta(0)$ and thus is computationally-efficient.

\section{Effects across Space (ANN Approach)}

Once the trend, daily, and hourly variations are accounted for in the model, there still exist some residuals in the Holt-Winter's model that are due to factors not accounted for in the model. Part of this can be explained as the influence of traffic conditions from one segment to another. For instance, if one segment is experiencing abnormally high travel time due to congestion, then it is likely to influence the traffic conditions on the next segment as well (depending upon the nature of traffic flow from one segment to the next) and will lead to higher-than-expected travel time on the next segment. However it must be noted that if there is no significant traffic flow from one segment to another across the bus route, then this correlation might be absent.

Mathematically, after controlling the observed data for the daily and hourly variations, the residuals from the model represent the noise due to correlation effects across space and the interaction of traffic streams across various segments. Taking a hint from the ANN approach, we can improve the quality of our forecast by including correlation effects between the residuals across different segments. Since the mean travel time across different segments varies depending upon the length of the segment, it is a good idea to carry out the above check after controlling for the above factor-that is, taking the residuals as a percentage of the expected travel times.

We define a corresponding Performance Factor $\left(P_{i}\right)$ for each segment $i$ at time $t$ expressing the residual from the model as a fraction of the expected value.

$$
P_{i, t}=\frac{u_{i, t}}{F_{i, t}}=1-\frac{D_{i, t}}{F_{i, t}}
$$


Where:

$$
\begin{aligned}
& u_{i, t}=\text { residual term from the model } \\
& D_{i, t}=\text { observed value } \\
& F_{i, t}=\text { forecasted (estimated) value }
\end{aligned}
$$

For each segment, we check for its correlation with the previous segment $i-1$ using the following hypothesis:

$$
P_{i, t}=k \cdot P_{i-1, t}
$$

Wherever the correlation is significant within the accepted significance level, the effect was considered and captured in the correlation factor $k$.

\section{Dwell Time}

Dwell times at a bus stop usually vary from a few seconds to less than a minute, with the average being less than half a minute. Thus, a rigorous time series analysis, as was done in the travel time data, is not required.

The approach that the model uses is to group the travel time into eight two-hour intervals, as done previously. Since the variations are fewer, a simple average of dwell times can be taken for each period to estimate the dwell time at different times of the day.

$$
t_{d w e l l, i}=\sum_{k=1}^{n} \frac{d_{i, k}}{n}
$$

\section{Performance Measures}

The study uses the following measures to evaluate the forecasts-the mean absolute error (MAE), the mean absolute percentage error (MAPE), and the root mean square error (RMS). The three terms can judge the difference between the observed and the predicted running time in different aspects.

- $\operatorname{MAE}=\frac{1}{N} \sum\left|t_{a}-t_{f}\right|$

- $\mathrm{MAPE}=\frac{1}{N} \sum \frac{\left|t_{a}-t_{f}\right|}{t_{a}} \times 100 \%$

- $\mathrm{RMSE}=\sqrt{\frac{\sum(t a-t f)^{2}}{N-1}}$

Where $t_{a}$ is the running time of the bus and $t_{f}$ is the forecasted (estimated) running time.

\section{Validation Results}

For the study, one month of GPS data were available for DTC Route 78, as discussed above. The route comprises 15 bus stops. For the study, three stops were omitted-Wazirpur Depot, Punjabi Bagh Depot, and Swatantra Mills-as their GPS coordinates were not clear from Google Maps.

Data extraction was carried out using MATLAB code, which was then put into a spreadsheet file. Holts Winter's Double Seasonal Model was applied separately to each segment. 
The parameters were optimized using the Solver add-in available in Excel using Generalized Reduced Gradient (GRG) ${ }^{2}$ method. The processed data matrix then was imported back into Excel, which was then used to arrive at the arrival time estimates for bus. The forecasting functions were developed in this way to use only the past data for predictions.

\section{Individual Segments}

Table 2 shows the results when the model was applied to different segments across the route along with the minimized error estimates. The linear part of the model estimates the increase in average travel time across the segment due to long-term changes in traffic conditions. Since the observation set has only one month of data, the parameters $\alpha, \beta$ are estimated to be zero, as no significant change in average traffic conditions was expected over one month. This implies that the average traffic for the month is constant. The linear parameters are estimated to be non-zero for the first segment. This might be due to permanent change in traffic conditions on the segment owing to construction/other obstructions on the segment. The parameters updating the daily and hourly factors have very low values. This represents the low effect of a single observation on the model definitions and more importance is given to the cumulative past information. This validates our assumption about consistency in traffic condition in the same state.

TABLE 2.

\begin{tabular}{|c|l|l|c|c|c|c|c|}
\hline Segment & \multicolumn{1}{|c|}{ Start Stop } & \multicolumn{1}{|c|}{ End Stop } & $\boldsymbol{\alpha}$ & $\boldsymbol{\beta}$ & $\boldsymbol{\lambda}$ & $\boldsymbol{\delta}$ & RMSE (min) \\
\hline 1 & Azadpur & Shalimar Bagh & 0.003 & 0.125 & 0.132 & 0.115 & 1.003 \\
\hline 2 & Shalimar Bagh & Ashok Vihar & 0.000 & 0.000 & 0.080 & 0.067 & 1.871 \\
\hline 3 & Ashok Vihar & PreambariPul & 0.000 & 0.000 & 0.130 & 0.068 & 0.476 \\
\hline 4 & PreambariPul & Brittania & 0.000 & 0.000 & 0.059 & 0.056 & 1.218 \\
\hline 5 & Brittania & Rampura & 0.000 & 0.000 & 0.201 & 0.133 & 1.568 \\
\hline 6 & Rampura & Zakhira & 0.000 & 0.000 & 0.056 & 0.125 & 0.651 \\
\hline 7 & Zakhira & Moti-Nagar & 0.000 & 0.000 & 0.146 & 0.079 & 1.277 \\
\hline 8 & Moti-Nagar & Shadipur Depot & 0.000 & 0.000 & 0.204 & 0.061 & 1.454 \\
\hline 9 & Shadipur Depot & Naraina Depot & 0.000 & 0.000 & 0.082 & 0.017 & 1.250 \\
\hline 10 & Naraina Depot & LohaMandi & 0.000 & 0.000 & 0.267 & 0.000 & 1.266 \\
\hline 11 & LohaMandi & Inderpuri & 0.000 & 0.000 & 0.300 & 0.138 & 1.341 \\
\hline
\end{tabular}

Using the above standard errors, following performance estimates were worked out for the model:

- Std. error for route: $4.22 \mathrm{~min}$

- Average total travel time: $42.8 \mathrm{~min}$

- Average error: $9.8 \%$

Figure 1 shows the typical observation vs. forecast trend for a given segment across time. It can be seen that in the initial periods, the errors were large, as the model was training. However, after multiple inputs, the quality of the forecasts improved with time.

\footnotetext{
2 The GRG Non-Linear method is a popular mathematical approach used to optimize multi-variable
} problems under multiple constraints and is available as an in-built feature with the Solver add-in. 
FIGURE 1.

Typical observation vs. forecast for a segment using Holt-Winter's model

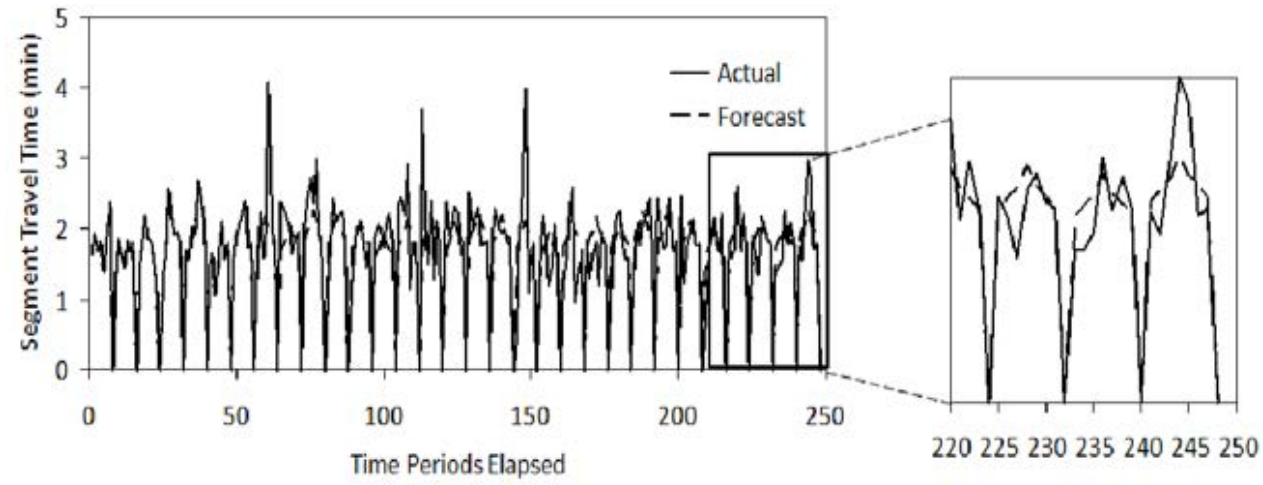

\section{Dwell Time}

Since the observed dwell times were usually small for different segments, it was decided to follow a stationary model superimposed with different seasonal factors for different times of the day. The seasonal factors were calculated by dividing each observation by the overall average of the data series, since we are assuming a stationary data series.

It was found that mean dwell time is less than half a minute for all the bus stops on the route. Thus, a simple model for the stationary series was used to predict the dwell time, as a few seconds' error in the model would not introduce many errors in the forecast.

\section{Correlation Effects}

After accounting for trend and seasonality in Holt-Winter's model, the residuals were tested for correlation to study the interaction between different segments. Thus, we defined appropriate performance factor for each observation, which were tested for correlation with the immediate predecessor.

Correspondingly, a regression was carried out and all the correlation factors within the significance level of $20 \%$ were accepted. The results are summarized in Table 3.

TABLE 3.

\section{\begin{tabular}{|c|c|c|c|c|c|} 
Seg. & Corr & T-Stat & Sig. (\%) & Inference & $\mathbf{k}$ \\
\hline
\end{tabular}}

Correlation across Segments

\begin{tabular}{|r|r|r|r|l|r|}
\hline $1-2$ & 0.18 & 1.68 & 9.33 & Sig. & 0.184 \\
\hline $2-3$ & 0.00 & 0.02 & 98.48 & Insig. & 0.000 \\
\hline $3-4$ & 0.07 & 0.83 & 40.89 & Insig. & 0.000 \\
\hline $4-5$ & -0.07 & -0.89 & 36.97 & Insig. & 0.000 \\
\hline $5-6$ & -0.089 & -1.13 & 25.82 & Insig. & 0.000 \\
\hline $6-7$ & 0.03 & 0.62 & 53.53 & Insig. & 0.000 \\
\hline $7-8$ & -0.05 & -0.49 & 62.20 & Insig. & 0.000 \\
\hline $8-9$ & 0.15 & 4.74 & 0.00 & Sig. & 0.154 \\
\hline $8-10$ & 0.33 & 3.75 & 0.02 & Sig. & 0.329 \\
\hline $10-11$ & 1.03 & 31.99 & 0.00 & Sig. & 1.026 \\
\hline
\end{tabular}

\section{Prediction along the Route}

Table 4 shows the cumulative error estimates as the bus travels across the route, and Figure 2 shows the evolution of the total error as the bus travels across the route. It should 
be noted that the cumulative error stays below the standard error predicted for the route, and the absolute percentage error also remains largely within the $10 \%$ limit.

TABLE 4.

Cumulative Error Estimates for Route on January 31, 2013, for Journey Starting at 2:15 PM

\begin{tabular}{|l|c|c|c|c|c|c|c|c|c|c|c|}
\hline \multicolumn{1}{|c|}{ Stop } & $\mathbf{1}$ & $\mathbf{2}$ & $\mathbf{3}$ & \multicolumn{1}{c|}{$\mathbf{5}$} & $\mathbf{5}$ & $\mathbf{6}$ & $\mathbf{7}$ & $\mathbf{8}$ & $\mathbf{9}$ & $\mathbf{1 0}$ & $\mathbf{1 1}$ \\
\hline Dist (km) & 1.3 & 1.7 & 2.45 & 4.85 & 10.85 & 12.05 & 14.89 & 16.69 & 17.54 & 18.54 & 19.14 \\
\hline Cumm. Error (min) & 0.19 & 0.46 & 0.29 & 2.75 & 1.07 & 1.38 & 1.14 & 0.60 & 0.68 & 1.46 & 1.66 \\
\hline MAE (min) & 0.19 & 0.43 & 0.34 & 0.87 & 1.46 & 1.27 & 1.12 & 1.05 & 1.07 & 1.05 & 0.97 \\
\hline MAPE (\%) & 5.75 & 7.17 & 3.37 & 26.24 & 6.03 & 6.69 & 4.79 & 2.36 & 2.41 & 4.85 & 5.16 \\
\hline RMSE (min) & 0.19 & 0.92 & 0.72 & 1.08 & 1.35 & 1.23 & 1.14 & 1.09 & 1.10 & 1.08 & 1.03 \\
\hline
\end{tabular}

FIGURE 2.

Real-time forecasting across whole route

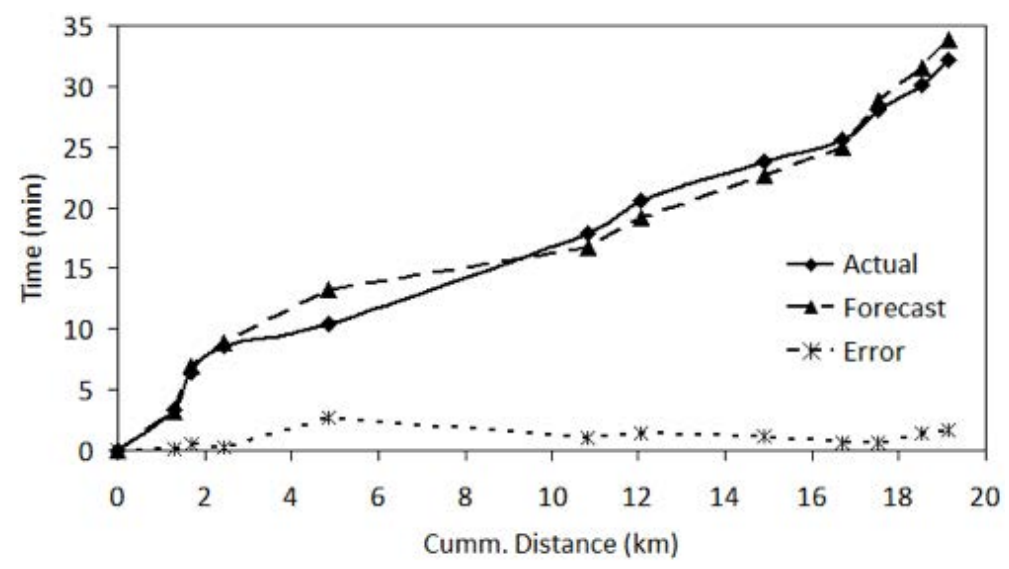

\section{Multiple Step Ahead Forecasts}

Table 5 contains the performance measures of the proposed model with the moving averages model (most commonly-used field method). For the moving average, three categories were used: Past Three Periods (MA3), Past One Day (MA8) and Past One Week (MA56; long-term average). Standard performance measures were calculated and compared for different models. To compare different models, Real-time, 5-day ahead, and 10-day ahead forecasts were developed for the bus travel times.

TABLE 5. Comparison of Different Algorithms for Multiple Step Ahead Forecasts for January 29, 2013

\begin{tabular}{|c|c|c|c|c|c|c|c|c|c|c|c|c|}
\hline & \multicolumn{3}{|c|}{ Proposed Model } & \multicolumn{3}{|c|}{ MA3 } & \multicolumn{3}{|c|}{$\begin{array}{c}\text { MA8 } \\
\text { (Past } 1 \text { Day Data) }\end{array}$} & \multicolumn{3}{|c|}{$\begin{array}{c}\text { MA56 } \\
\text { (Past } 1 \text { Week Data) }\end{array}$} \\
\hline & Real-Time & 5-day & 10-day & Real-Time & 5-day & 10-day & Real-Time & 5-day & 10-day & Real-Time & 5-day & 10-day \\
\hline Actual Obs. (min) & 38.81 & 38.81 & 38.81 & 38.81 & 38.81 & 38.81 & 38.81 & 38.81 & 38.81 & 38.81 & 38.81 & 38.81 \\
\hline Forecast (min) & 39.88 & 38.03 & 39.06 & 39.06 & 39.90 & 36.30 & 33.94 & 33.81 & 33.00 & 33.31 & 33.00 & 33.24 \\
\hline MAE & 1.07 & 0.78 & 0.25 & 0.25 & 1.09 & 2.51 & 4.87 & 5.00 & 5.81 & 5.50 & 5.81 & 5.57 \\
\hline MAPE & 2.75 & 2.02 & 0.63 & 0.63 & 2.80 & 6.48 & 12.56 & 12.89 & 14.98 & 14.18 & 14.98 & 14.36 \\
\hline
\end{tabular}

${ }^{3}$ The value of $10 \%$ on MAPE is the expected value of MAPE in the general case. The value of $26 \%$ is for one particular instance of the algorithm in which distortions in travel time could have been caused by unforeseen circumstances on the concerned segment. This is a one-time random error that is associated with that particular instant at that segment. 
Figure 3 graphically compares the performance of the two models by plotting the percentage error estimates. It can be seen that the performance of the current algorithm is consistently better than of the commonly-used methods with significantly fewer errors.

FIGURE 3.

Percentage error for different algorithms

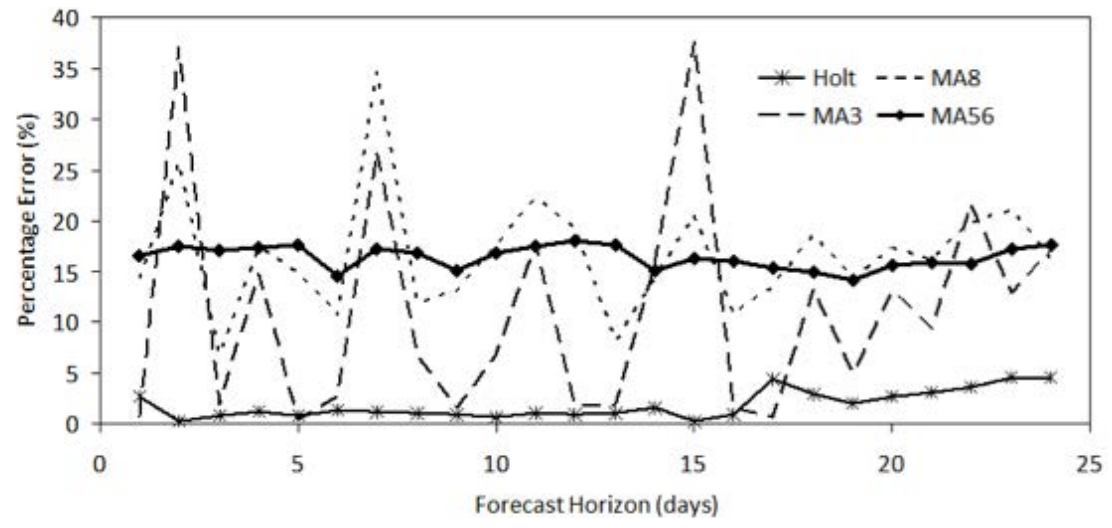

\section{Different Times of the Day}

Table 6 shows how the model performed at different times of the day. It can be seen in Figure 4 that the errors lie within the range that was predicted and, thus, the model is successfully able to account for variations arising due to different times of the day.

TABLE 6.

Performance on Different Times of Day on January 29,

\begin{tabular}{|l|r|r|r|r|r|r|r|}
\hline & 8:00 AM & 10:00 AM & 12:00 PM & 2:00 PM & 4:00 PM & 6:00 PM & 8:00 PM \\
\hline Actual (min) & 36.37 & 47.01 & 38.87 & 47.71 & 35.20 & 36.60 & 29.52 \\
\hline Forecast (min) & 40.00 & 44.59 & 41.70 & 43.60 & 36.34 & 39.53 & 33.47 \\
\hline MAE (min) & 3.63 & 2.42 & 2.83 & 4.10 & 1.14 & 2.92 & 3.96 \\
\hline
\end{tabular}

FIGURE 4.

Performance at different times of day

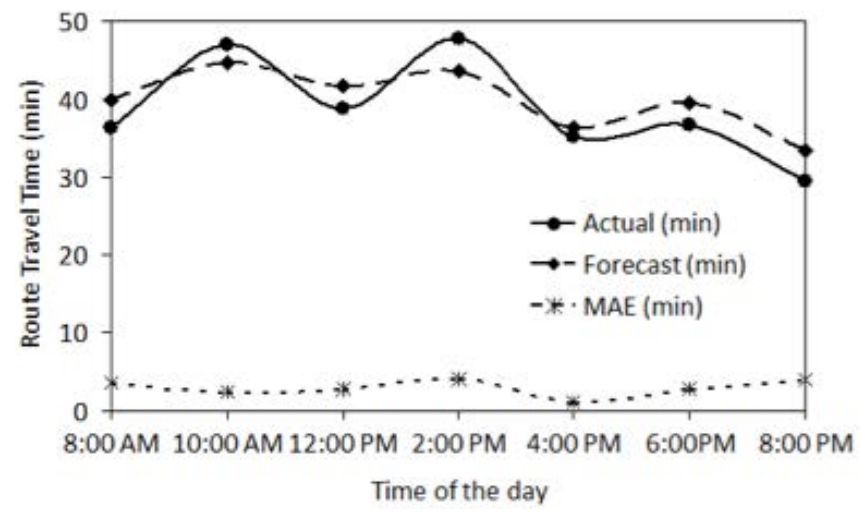

\section{Implementation}

The predicted travel time obtained from the proposed algorithm can be easily formatted to suit any mode of arrival time information displays such as at bus stops, within bus, or through websites. 
The decision of an appropriate display depends on the requirements of the end user. For example, a passenger standing at a bus stop would be interested in knowing about the arrival time of the next bus in a particular route, whereas a passenger traveling on a bus would be interested in knowing about the arrival time of that bus at a specific bus stop. A sample display, which conforms to these requirements, has been generated for the current study and is shown below.

- At the bus stop: Current time - 2:04 PM

Arrival time bus $78-2: 13$ PM

- Inside the bus: Current time - 2:04 PM

Estimated time of arrival:

- Rampura - 2: 13 PM

- Zakhira - 2: 17 PM

- Moti Nagar - 2: 21 PM

- Shadipur Depot - 2: 24 PM

Similarly, the results from the model can be incorporated into different forms of inquiry systems and can cater to a wide range of end users through different media.

\section{Conclusions}

The present study developed a model-based algorithm for real-time automated bus arrival prediction, taking into account the time and space variations involved during travel. The main contributions of this study include automated travel time prediction, explicitly incorporating information about seasonality in the data series (time of day and day of week) and real-time updating using GPS data from the buses, thus making it possible to incorporate the changes in the field in real time. The prediction scheme used the Double Seasonal Holt-Winter's Exponential Smoothing technique to predict the travel time of buses, which keeps track of real-time parameters at four different levels in the data series and is able to account for time-trend and seasonality in the data series. The proposed model also checked for correlation in the residuals from the model to account for space effects in the data series different segments.

The results show that the proposed model is able to estimate the travel times within an accuracy of approximately $10 \%$, which is better than the traditionally-used elementary moving average models. The incorporation of real-time data and the complete automation make the proposed scheme ready for field implementation. This scheme can be adopted for real-time bus arrival time prediction under Indian conditions for reliable PIS implementation.

\section{Acknowledgments}

The authors acknowledge the support of Dr. Manika Aggarwal from DIMTS Delhi and Prof. Geetam Tiwari from IIT Delhi for providing the required bus GPS data in this study. 


\section{References}

Baker, C. B., and A. C. Nied. Predicting bus arrival using One Bus Away real-time data. http://homes.cs.washington.edu/ anied/papers/AConradNied_OneBusAway_ Writeup_20131209.pdf. Accessed November 2014.

Biagioni, J., T. Gerlich, T. Merrifield, and J. Eriksson. 2011. Easy Tracker: Automatic transit tracking, mapping, and arrival time prediction using smartphones. SenSys'11, November 2011, Seattle, WA.

Bin, Y. 2006. Bus arrival time prediction using support vector machines. Journal of Intelligent Transportation Systems, 10(4): 151-158.

Cathey, F. W., and D. J. Dailey. 2003. A prescription for transit arrival/departure prediction using automatic vehicle location data. Transportation Research Part C, 11(3): 241-264.

Chang, H., D. Park, S. Lee, H. Lee, and S. Baek. 2010. Dynamic multi-interval bus travel time prediction using bus transit data. Transportmetrica, 6(1): 19-38.

Chen, M., X. Liu, J. Xia, and S. I. Chien. 2004. A dynamic bus arrival time prediction model based on APC data. Computer-Aided Civil and Infrastructure Engineering, 19(5): 364376.

Chien, S. I. J., and C. M. Kuchipudi. 2003. Dynamic travel time prediction with real-time and historic data. Journal of Transportation Engineering-ASCE, 129(6): 608-616.

Chien, S. I. J., Y. Ding, and C. Wei. 2002. Dynamic bus arrival time prediction with artificial neural networks. Journal of Transportation Engineering-ASCE, 128(5): 429-438.

Cristianini, N., and J. Shawe-Taylor. 2000. An Introduction to Support Vector Machines and Other Kernel-based Learning Methods. Cambridge University Press, Cambridge, UK.

Jeong, R., and L. R. Rilett. 2004. Bus arrival time prediction using artificial neural network model. 7th International IEEE Conference on Intelligent Transportation Systems 2004, Washington DC.

Li Z., H. Yu, Y. Liu, and F. Liu. 2008. An improved adaptive exponential smoothing model for short-term travel time forecasting of urban arterial street. Acta Automatica Sinica, 34: 1404-1409.

Liu, X. S. I. Chien, and M. Chen. 2012. An adaptive model for highway travel time prediction. Journal of Advanced Transportation, 46. DOI: 10.1002/atr.1216.

Mazloumi, E., G. Currie, G. Rose, and M. Sarvi. 2009. Using scats data to predict bus travel time. http://atrf.info/papers/2009/2009_Mazloumi_Currie_Rose_Sarvi.pdf. Accessed November 2014.

Miranda, C., R. C. Souza, M. Barros, and M. De Miranda. 2007. Short term load forecasting using double seasonal exponential smoothing and interventions to account for holidays and temperature effects. Pontifícia Universida de Católica do Rio de Janeiro, Brazil. http://www.ecomod.net/sites/default/files/document-conference/ ecomod2007/294.pdf, accessed May 2013. 
Padmanaban, R. P. S., K. Divakar, L. Vanajakshi, and S.C. Subramanian. 2010. Development of a real-time bus arrival prediction system for Indian traffic conditions. IET Intelligent Transport Systems 4(3): 189-200.

Park, S. H., Y. J. Jeong, and T. J. Kim. 2007. Transit travel time forecasts for location-based queries: Implementation and evaluation. Journal of the Eastern Asia Society for Transportation Studies, 7: 1859-1869.

Ramakrishna Y., P. Ramakrishna, V. Lakshmanan, and R. Sivanandan. 2006. Bus travel time prediction using GPS data. http://www.gisdevelopment.net/proceedings/ mapindia/2006/student\%20oral/mi06stu_84.htm, accessed March 2013.

Richman, M. S., and T. W. Parks. 1998. Universal trainable detection and arrival time estimation methods using support vector machines. http://citeseerx.ist.psu.edu/ viewdoc/summary?doi=10.1.1.52.7201. Accessed November 2014.

Shalaby, A., and A. Farhan. 2004. Prediction models of bus arrival and departure times using AVL and APC data. Journal of Public Transportation, 7(1): 41-61.

Smith, B. L., B. M. Williams, and R.K. Oswald. 2002. Comparison of parametric and nonparametric models for traffic flow forecasting. Transportation Research Part C, 10(4): 303-321.

Son, B. H. Y. Kim, C. Y. Shin, and S. K. Lee. 2004. Bus arrival time prediction method for ITS application. Knowledge-Based Intelligent Information and Engineering Systems, Lecture Notes in Computer Science, 3215: 88-94

Vapnik, V. N. 1999. An overview of statistical learning theory. IEEE Transactions on Neural Networks, 10(5): 988-999.

Vapnik, V. N. 2000. The Nature of Statistical Learning Theory. Springer, New York.

Vanajakshi, L., S. Subramanian, and R. Sivanandan. 2009. Travel time prediction under heterogeneous traffic conditions using global positioning system data from buses. IET Intelligent Transport Systems, 3(1): 1-9.

You, J. S., and T. J. Kim. 2000. Development and evaluation of a hybrid travel time forecasting model. Transportation Research Part C, 8(1-6): 231-256.

Yu, B., W. H. K. Lam, and M. T. Tam. 2011. Bus arrival time prediction at bus stop with multiple routes. Transportation Research Part C, 19(6): 1157-1170.

Yu, B., Z. Z. Yang, and B. Z. Yao. 2006. Bus arrival time prediction using support vector machines. Journal of Intelligent Transportation Systems, 10(4): 151-158.

Yu, B., Z. Z. Yang, and J. Wang. 2010. Bus travel-time prediction based on bus speed. Proceedings of the Institution of Civil Engineers-Transport, 163(1): 3-7.

Yu, B., Z. Z. Yang, K. Chen, and B. Yu. 2010. A hybrid model for bus arrival time prediction. Journal of Advanced Transportation, 44(3): 193-204.

Zhang, X. Y., and J. A. Rice. 2003. Short-term travel time prediction. Transportation Research Part C, 11(3-4): 187-210. 
Zhou, P., Y. Zheng, and L. Mo. 2014. How long to wait-Predicting bus arrival time with mobile phone. IEEE Transactions On Mobile Computing, 13(6): 1228-1241.

\section{About the Authors}

Prakhar Balasubramanian (prakhar.iitd@gmail.com) is a former undergraduate student in Civil Engineering at the Indian Institute of Technology Delhi. His main interests are in forecasting methods applied to transportation. He is currently working at a leading management consulting firm based out of Chicago.

K. RAmachandra RAo (rrkalaga@civil.iitd.ac.in) is an Associate Professor in Civil Engineering and Transportation Research and Injury Prevention Programme (TRIPP) at the Indian Institute of Technology Delhi. His main research interests are traffic modelling, public transit planning, road safety and low carbon mobility planning. He has worked earlier with Indian Institute of Technology Guwahati and University of Kwazulu-Natal, Durban. His present focus is on characterizing and developing appropriate models for heterogeneous traffic. He has authored several articles published in various scholarly journals. 